\title{
Face Detection
}

\author{
Amir Nobahar Sadeghi Nam \\ Post Doc. Research Fellow, Department of Mechatronics Engineering \\ Atilim University, Ankara, Turkey
}

\begin{abstract}
Face detection is one of the challenging problems in the image processing, as a main part of automatic face recognition. Employing the color and image segmentation procedures, a simple and effective algorithm is presented to detect human faces on the input image. To evaluate the performance, the results of the proposed methodology is compared with ViolaJones face detection method.
\end{abstract}

Keywords:- Component; Face Detection, Color Segmentation, Image Segmentation.

\section{INTRODUCTION}

Face detection is procedure of recognition human faces with different sizes in an image. It has many applications such as financial transactions, monitoring systems, credit card verification, automated teller machine access, personal computer access, video surveillance etc. Since the human face is a dynamic object with a high degree of appearance variability, so face detection is a challenging field in computer vision. A wide variety of methods and techniques have been presented in this subject. Some of them are based on principal feature or component analysis, some propose template matching. Color analysis, Hough transform, and neural network are the other employed techniques to detect human faces in the input image.

A face detection framework is presented in [1], which can detect faces with high rate and fast. The framework has three contributions, integral image to fast computation of the features, AdaBoost learning algorithm to select a small number of critical visual features, and cascade classifiers to discard background of the image. Paper [2], presents algorithms subjected as feature-based and image-based, and then discusses their technical approach and performance. Also some proposed applications are presented in this paper. A face detection algorithm for color images is presented in [3]. The presented method is capable of detecting faces on an image with varying lighting conditions and complex backgrounds. It detects faces based on the skin color and then constructs eye, mouth, and boundaries for verifying each face candidate. Article [4], presents a neural network-based face detection system. The employed network accomplish the task with dividing the image to small windows and then examines whether each window has a candidate face. In [5], a shape comparison approach is proposed to detect human face fast, accurate and robust. The presented method is edge-base, which employs Hausdorff distance to measure similarity between a general face model and the objects on the image. In order to handle profile views and rotated faces, paper [6] implements extension of the Viola-Jones face detection framework. This framework has a slow training rate, but fast detection rate. Different detectors for different views of the face are built and then a decision tree is trained to determine the viewpoint class for the given image. Study [7], proposes an architecture to locate human faces in a complex background. The presented method is designed based on hierarchical knowledge-based method, which has three levels. Mosaic images at different resolutions, form the higher two levels and an improved edge detection method is proposed for the lower level. As illumination sensitivity influences face detection results, so selection of proper color space is important to detect objects on an image. Employing a proper color space for skin-color detection is studied in [8] and some of the methods in this regard are researched. A cascade architecture is proposed in [9], which is built on convolutional neural networks. The proposed cascade network operates at multiple resolutions, quickly rejects the background regions and carefully evaluates a small number of face candidates. Article [10] proposes a feature-based architecture to detect human face, which can be easily extended to handle more variations of the imaging conditions. The presented method detects feature points employing spatial filters and then groups them into face candidates by geometric and gray level constraints. To detect objects on an image, illumination invariant local structure features is introduced, which is designed based on a modified census transform and then an efficient four-stage classifier for rapid detection is presented in [11]. The proposed classifier is linear classifier, consisting of a set of feature lookup-tables. A novel face detection approach is presented in [12], which is based on a convolutional neural architecture. The proposed method is able to detect rotated and turned up faces in complex real world images. Article [13], presents a selfadaptive face detection algorithm based on skin color for images with complex background. First histogram skin color model is constructed and then skin color segmentation is implemented employing histogram back projection. To make further optimization of the segmentation, morphological and blob analysis are employed. A novel method to detect human faces in color images under non-constrained scene conditions, $\mathrm{s}$ is proposed in [14]. First color clustering and filtering using $\mathrm{YCbCr}$ and HSV approximations of the skin color are applied on the original image, then a merging stage is performed on the set of homogeneous skin color regions. Face constraints related to shape and size are considered, and then employing a wavelet packet decomposition, intensity texture is analyzed to detect faces. In [15], an approach to detect human faces three-dimensionally is proposed which combines a feature-based method with a holistic one. First, eyes and nose are detected employing the curvature of the surface analysis. Then the achieved 
result is processed by a PCA-based classifier trained to discriminate between faces and non-faces.

In this paper to detect human faces on the input image, a simple and effective architecture is presented based on the color and image segmentation methods. To evaluate its performance, the results of the proposed methodology is compared with well-known Viola-Jones face detection method.

\section{DETECTION ARCHITECTURE}

Detection of skin color in color images is a very popular and useful technique for face detection. While the input color image is typically in the RGB (Red, Green, Blue) format, the face detection techniques usually use color components in the color space, such as the HSV (Hue, Saturation, Value) or YIQ (Y: Luminance, I and Q: Chrominance Information) formats. That is because RGB components are subject to the lighting conditions thus the face detection may fail if the lighting condition changes. Among the color spaces, $\mathrm{YCbCr}$ components is employed in this study. In these components, the luminance information is contained in $\mathrm{Y}$ component; and, the chrominance information is in $\mathrm{Cb}$ and $\mathrm{Cr}$. Therefore, the luminance information can be easily de-embedded. The $R G B$ components are converted to the $\mathrm{YCbCr}$ components using the equations (1-3). The primary sample image, the converted $\mathrm{YCbCr}$ image, and its histogram distribution are shown in figures 1, 2 and 3. It is worth mentioning that the input image should be captured under almost same illuminate condition.

$$
\begin{aligned}
& Y=0.299 R+0.587 G+0.114 B \\
& C b=-0.169 R-0.332 G+0.500 B \\
& C r=0.500 R-0.419-0.081 B
\end{aligned}
$$

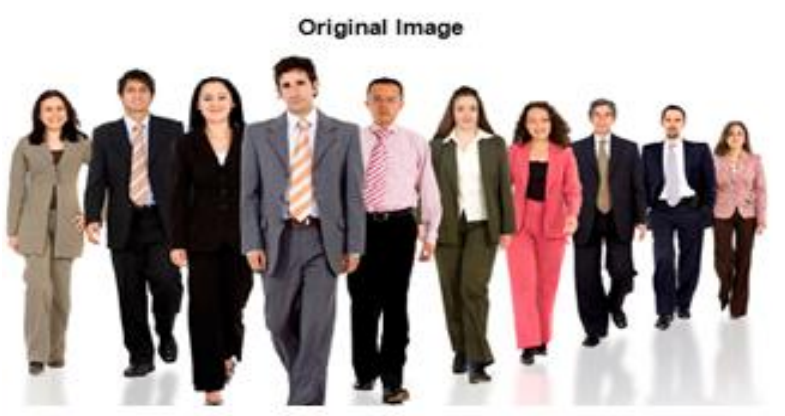

Fig 1:- Original $R G B$ Sample Image

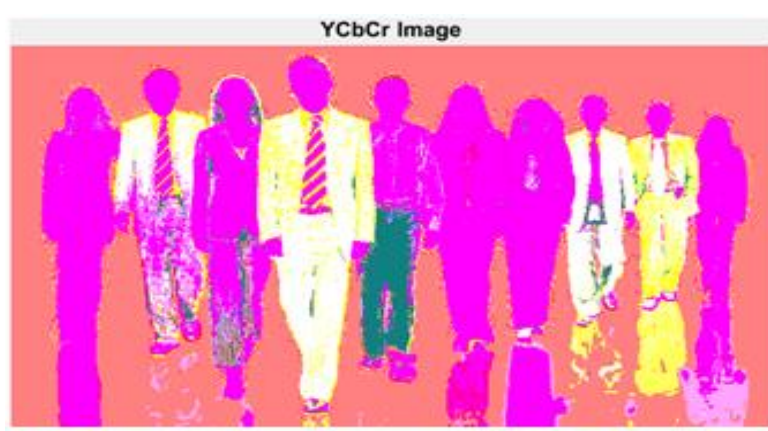

Fig 2:- $\mathrm{YCbCr}$ Image

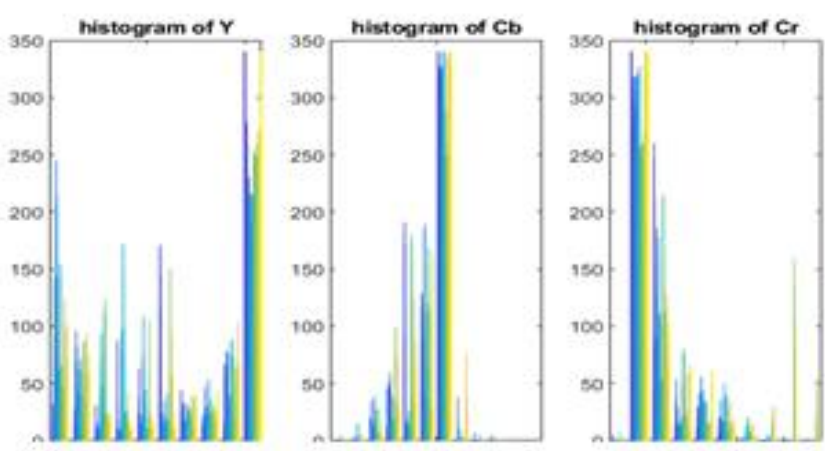

Fig 3:- Histogram distribution of $\mathrm{Y}, \mathrm{Cb}$, and $\mathrm{Cr}$

The presented procedure to detect faces on an image in this architecture, is based on color segmentation and then image segmentation. The color segmentation phase is accomplished to detect the skin colors, in which each pixel is classified as skin or non-skin, based on its color components. Figure 4 illustrates the result of color segmentation. As it seen, some non-skin objects are observed as their colors fall into the skin color space. This problem is inevitable, which should be improved.

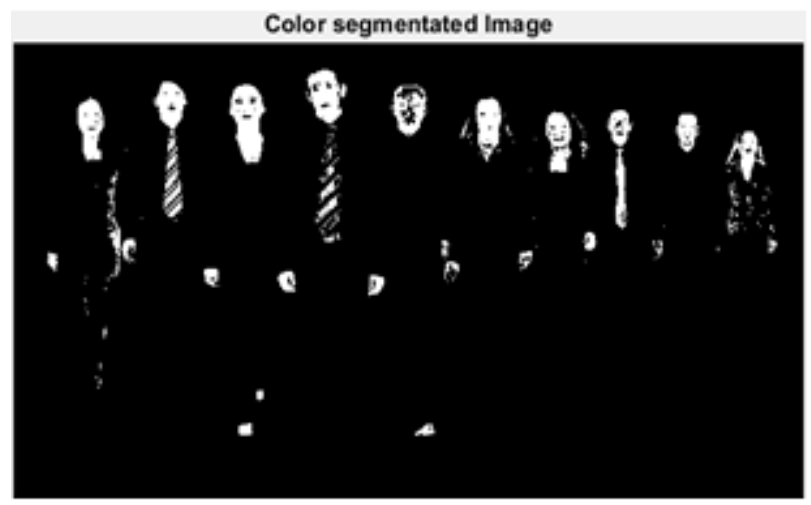

Fig 4:- Color Segmented Image

The Image segmentation phase, is started to separate the image blobs in the last binary image into individual regions. The process consists of three steps. First the black isolated holes are filled up, then the white isolated regions are removed, which are smaller than a predefined minimum face area, and finally the achieved image is eroded. Erosion is one of two fundamental morphological operations, which removes pixels on object boundaries. Figures 5, 6, and 7 display results of these three steps.

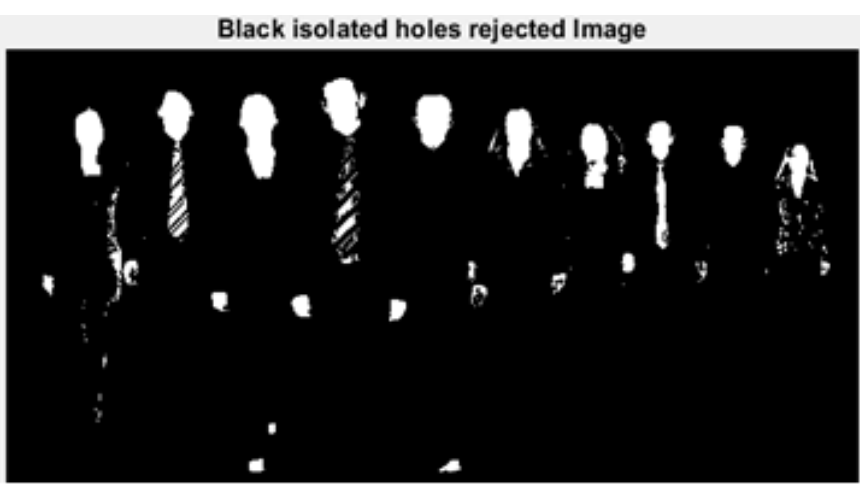

Fig 5:- Black Isolated Holes Rejected Image 


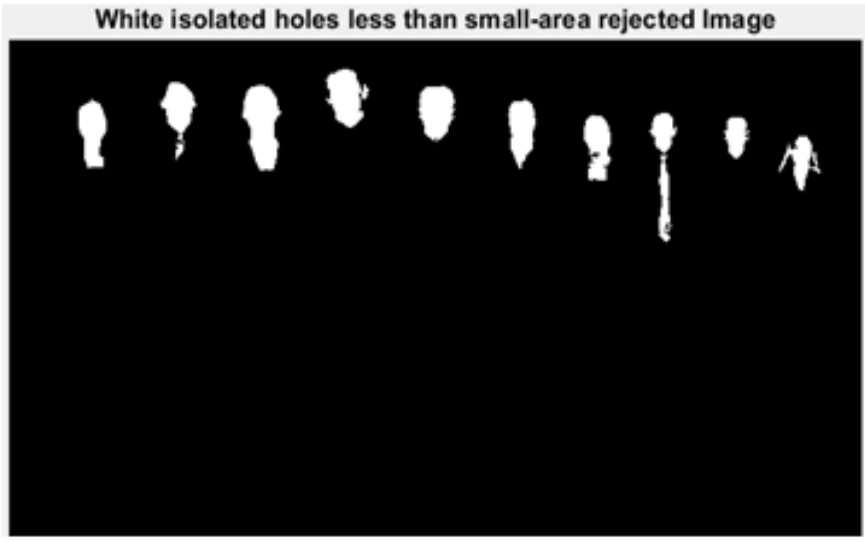

Fig 6:- White Isolated Holes Removed Image

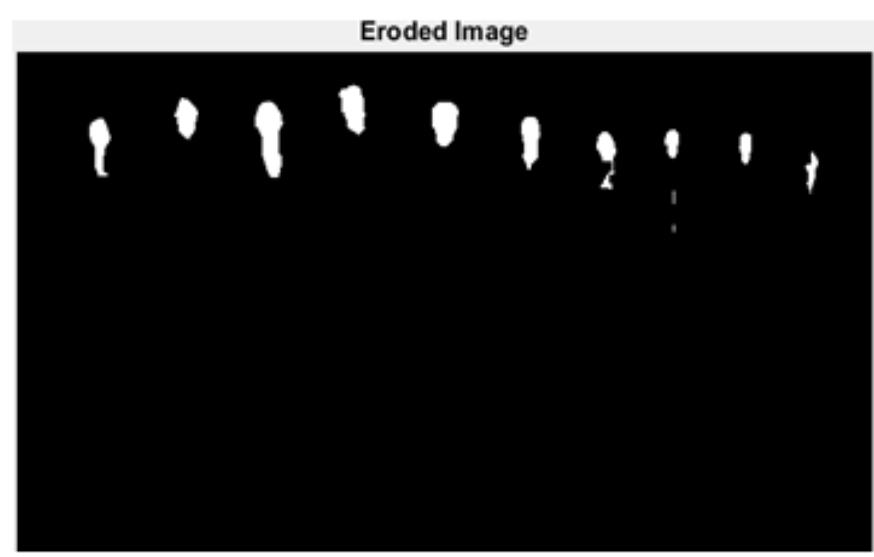

Fig 7:- Eroded Image

Secondly, to separate some integrated regions into individual faces, the Roberts-Cross edge detection algorithm is applied on the last image. This operator highlights regions of high spatial gradients that often correspond to edges. The highlighted region is converted into black lines and eroded to connect crossly separated pixels, as shown in the figure 8 . Finally, the eroded image and edge image (figures 7 and 8) are integrated into one binary image (figure 9) and relatively small black and white areas are removed. The difference between this process and the initial small area elimination is that the edges connected to black areas remain even after filtering. And those edges play important roles as boundaries between face areas after erosion. One more time, erosion, remove of small white and black isolated regions are repeated, which concludes to have an image shown in the figure 10.

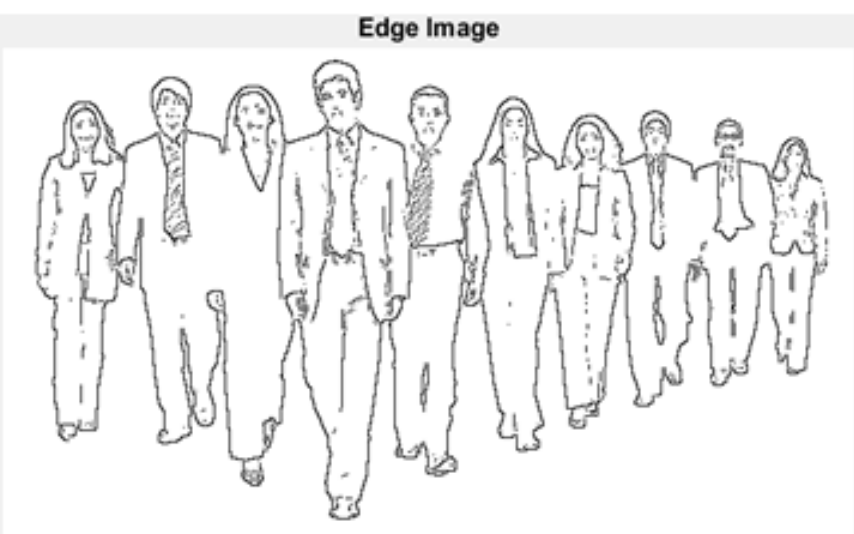

Fig 8:- Edges Detected by Roberts-Cross Operator

In order to detect exact location of faces with a square shape, previous processes are followed by an image matching process, which a set of Eigen-images are generated using the original image. Fig. 11, shows the final detected faces in the original image. These procedures are implemented on the second and third crowded original images. The results are relatively satisficed as shown in figures 12 and 13 .

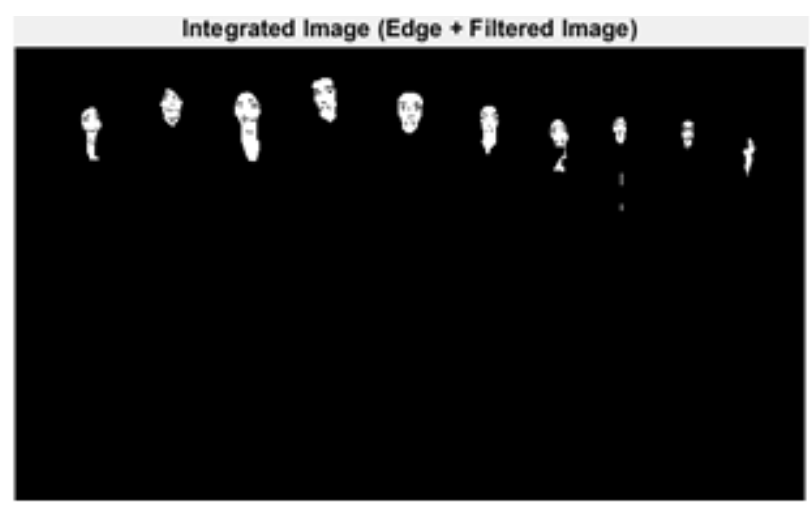

Fig 9:- Primary Integrated Binary Image

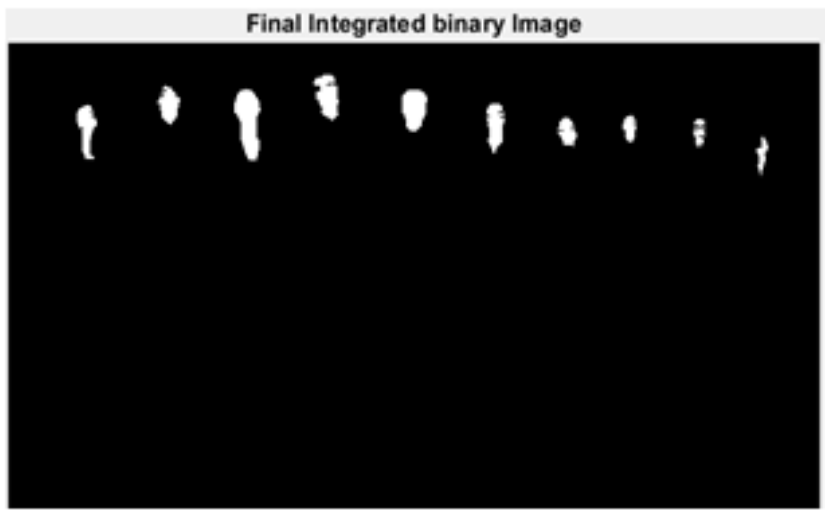

Fig 10:- Final Integrated Binary Image 


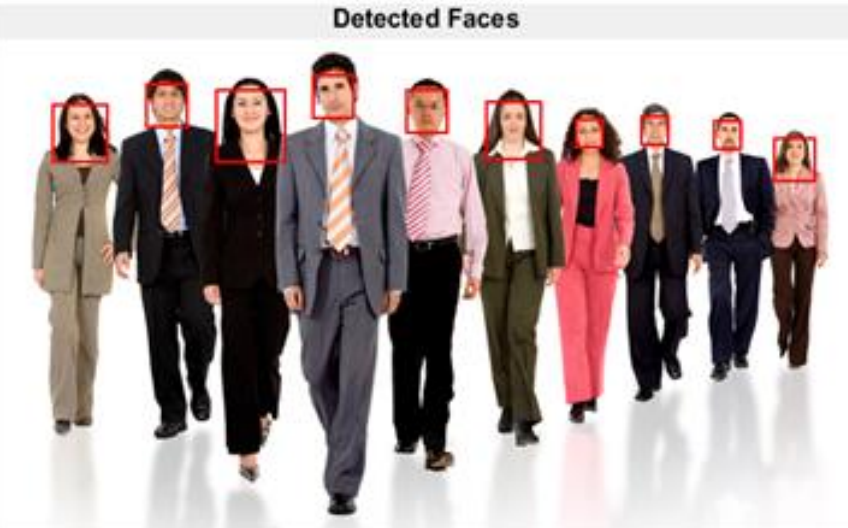

Fig 11:- Detected Faces on the First Sample Image

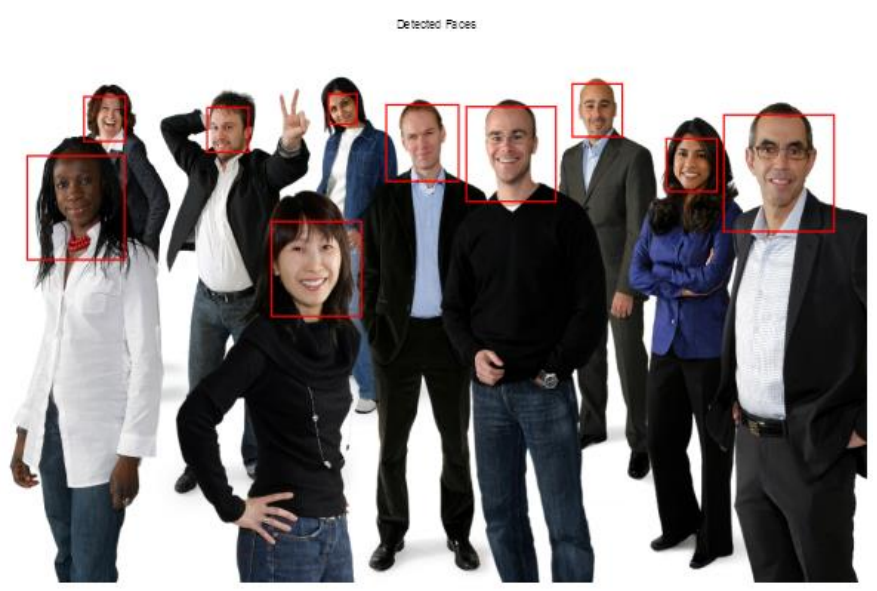

Fig 12:- Detected Faces on the Second Sample Image

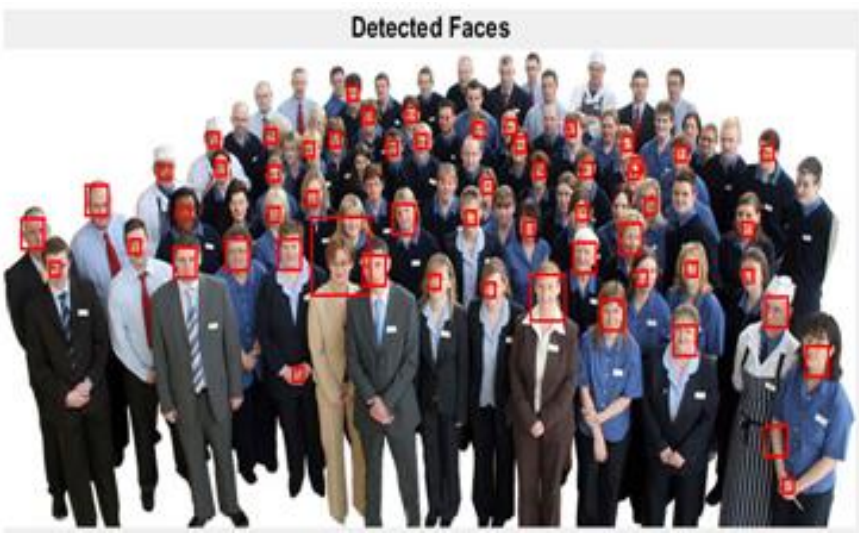

Fig 13:- Detected Faces on the Third Crowded Sample Image

In continue of the study, the Viola-Jones object detection algorithm is evaluated to detect face on the images. Robust and real-time are two main characteristics of this algorithm. It has four stages: Haar feature selection, creating an integral image, Adaboost training, cascading classifiers [16]. Figures 14, 15, and 16 display the detected faces in the first, second and third sample images.

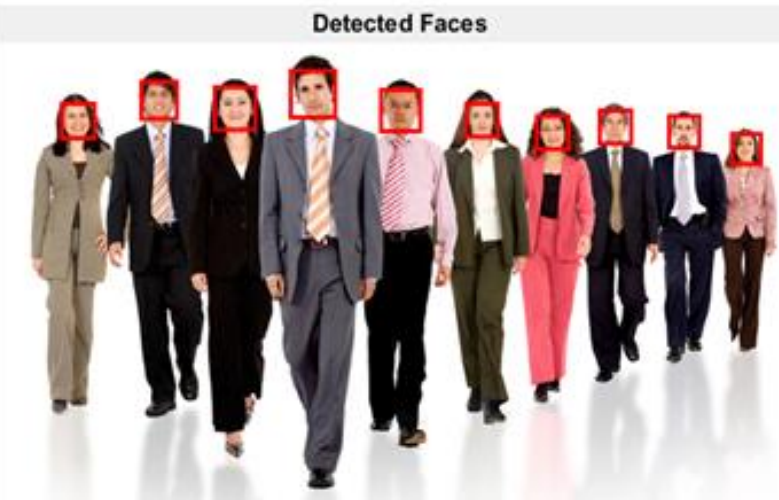

Fig 14:- Detected Faces on the First Sample Image by Viola-Jones Algorithm

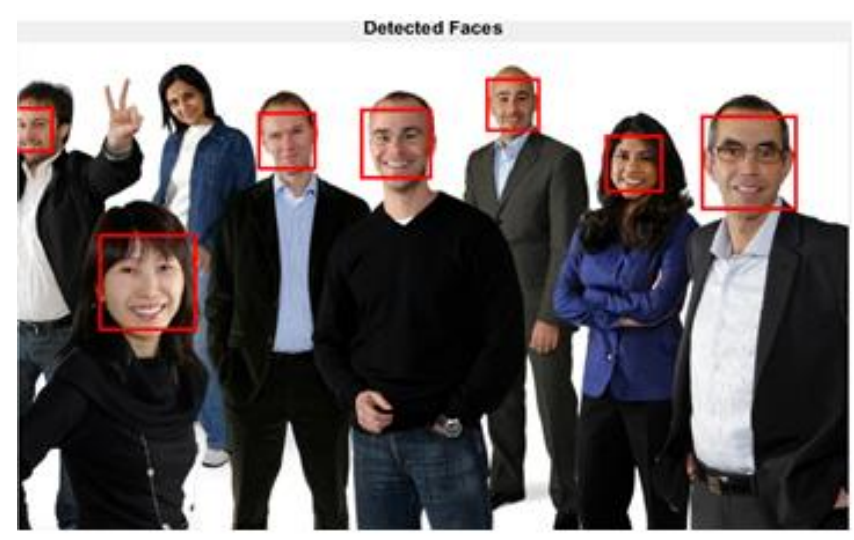

Fig 15:- Detected Faces on the Second Sample Image by Viola-Jones Algorithm

Some issues can be extracted from the resulted images of both algorithms. In the first sample image both of proposed algorithm and Viola-Jones algorithm detected $100 \%$ of total faces. In the second sample image, it is $100 \%$ for the proposed algorithm and $87.5 \%$ for Viola-Jones algorithm. The percentage of the detected faces decreases in the third sample image to $70.5 \%$ for the proposed algorithm and to $30.5 \%$ for Viola-Jones algorithm. The running time of each algorithm is measured for each of sample images, which are illustrated in the table 1 . The proposed method yields good results in face detection and maintains the speed advantage.

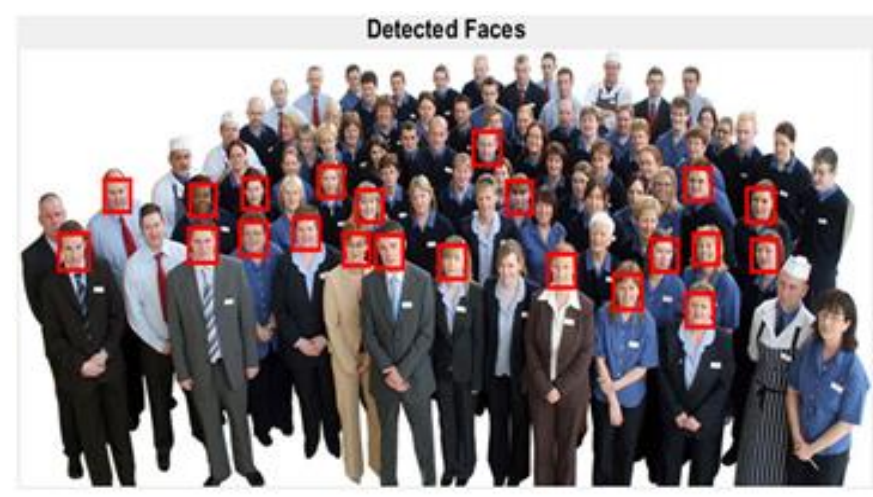

Fig 16:- Detected Faces on the Third Crowded Sample Image by Viola-Jones Algorithm 


\begin{tabular}{|c|c|c|}
\hline $\begin{array}{c}\text { Sample } \\
\text { Image }\end{array}$ & Proposed Algorithm & $\begin{array}{c}\text { Viola-Jones } \\
\text { Algorithm }\end{array}$ \\
\hline 1 & $0.26 \mathrm{sec}$. & $0.27 \mathrm{sec}$. \\
\hline 2 & $0.71 \mathrm{sec}$. & $1.88 \mathrm{sec}$. \\
\hline 3 & $0.28 \mathrm{sec}$. & $0.29 \mathrm{sec}$. \\
\hline
\end{tabular}

Table 1:- Running Times of the Algorithms

\section{CONCLUSION}

Based on the color and image segmentation methods, a simple and effective algorithm was presented to detect human faces on the input image. The results of the proposed methodology was compared with Viola-Jones face detection method, to evaluate the performance of it. Although the proposed method has a simple algorithm, its ability to detect more faces on the image is much higher. Along with this advantage, it is strictly comparable in terms of the algorithm execution time.

\section{REFERENCES}

[1]. Viola, Paul, and Michael J. Jones. "Robust real-time face detection." International journal of computer vision 57.2 (2004): 137-154.

[2]. Hjelmås, Erik, and Boon Kee Low. "Face detection: A survey." Computer vision and image understanding 83.3 (2001): 236-274.

[3]. Hsu, Rein-Lien, Mohamed Abdel-Mottaleb, and Anil K. Jain. "Face detection in color images." IEEE transactions on pattern analysis and machine intelligence 24.5 (2002): 696-706.

[4]. Rowley, Henry A., Shumeet Baluja, and Takeo Kanade. "Neural network-based face detection." IEEE Transactions on pattern analysis and machine intelligence 20.1 (1998): 23-38.

[5]. Jesorsky, Oliver, Klaus J. Kirchberg, and Robert W. Frischholz. "Robust face detection using the hausdorff distance." International conference on audio-and video-based biometric person authentication. Springer, Berlin, Heidelberg, 2001.

[6]. Jones, Michael, and Paul Viola. "Fast multi-view face detection." Mitsubishi Electric Research Lab TR20003-96 3.14 (2003): 2.

[7]. Yang, Guangzheng, and Thomas S. Huang. "Human face detection in a complex background." Pattern recognition 27.1 (1994): 53-63.

[8]. Book: Kovac, Jure, Peter Peer, and Franc Solina.Human skin color clustering for face detection. Vol. 2. IEEE, 2003.

[9]. Li, Haoxiang, et al. "A convolutional neural network cascade for face detection." Proceedings of the IEEE conference on computer vision and pattern recognition. 2015.

[10]. Yow, Kin Choong, and Roberto Cipolla. "Featurebased human face detection." Image and vision computing 15.9 (1997): 713-735.
[11]. Froba, Bernhard, and Andreas Ernst. "Face detection with the modified census transform." Sixth IEEE International Conference on Automatic Face and Gesture Recognition, 2004. Proceedings.. IEEE, 2004.

[12]. Garcia, Christophe, and Manolis Delakis. "Convolutional face finder: A neural architecture for fast and robust face detection." IEEE Transactions on pattern analysis and machine intelligence 26.11 (2004): 1408-1423.

[13]. Liu, Qiong, and Guang-zheng Peng. "A robust skin color based face detection algorithm." 2010 2nd International Asia Conference on Informatics in Control, Automation and Robotics (CAR 2010). Vol. 2. IEEE, 2010.

[14]. Garcia, Christophe, and George Tziritas. "Face detection using quantized skin color regions merging and wavelet packet analysis." IEEE Transactions on multimedia 1.3 (1999): 264-277.

[15]. Colombo, Alessandro, Claudio Cusano, and Raimondo Schettini. "3D face detection using curvature analysis." Pattern recognition 39.3 (2006): 444-455.

[16]. Viola-Jones object detection framework, WIKIPEDIA, https://en.wikipedia.org/wiki/Viola\%E2\%80\%93Jones _object_detection_framework (Visited Date; 10.09.2020) 